\title{
SOLAR-WIND KRYPTON AND SOLID/GAS FRACTIONATION IN THE EARLY SOLAR NEBLLA
}

\author{
Roger C. Wiens and D. S. Burnett \\ Division of Geological and Planetary Sciences, California Institute of Technology \\ M. Neugebauer \\ Jet Propulsion Laboratory, California Institute of Technology \\ R. O. Pepin \\ School of Physics and Astronomy, University of Minnesota, Minneapolis
}

\begin{abstract}
Krypton is the best candidate for determining limits on solid/gas fractionation in the early sun because of the smoothness of the odd-mass abundance curve in its mass region, which permits relatively precise interpolations of its abundance assuming no fractionation. Here we calculate the solar-system $\mathrm{Kr}$ abundance from solar-wind noble-gas ratios, determined previously by lowtemperature oxidations of lunar ilmenite grains, normalized to Si by spacecraft solar-wind measurements. The estimated ${ }^{83} \mathrm{Kr}$ abundance of $4.1 \pm 1.5$ per $10^{6} \mathrm{Si}$ atoms is within uncertainty of estimates assuming no fractionation, determined from CI-chondrite abundances of surrounding elements. This is significant because it is the first such constraint on solid/gas fractionation, though the large uncertainty only confines it to somewhat less than a factor of two.
\end{abstract}

\section{Chemical Fractionation in the Proto-Sun}

Based on astrophysical observations it is widely accepted that the solar system formed from an interstellar cloud that was initially a multi-phase system consisting of gas plus various interstellar grain components. Until temperatures reached the order of $2 \times 10^{3} \mathrm{~K}$, this multiphase nature must have been present throughout the formation of the sun and the planetary objects. A priori it may be unreasonable to assume that the formation of the sun and the solar nebula incorporated these phases in exactly the same relative proportions as in the initial interstellar cloud. The cloud itself was undoubtedly inhomogeneous on small scales, but the degree of inhomogeneity on large scales, of the order of AU, is totally unknown. Differential forces (e.g., magnetic) on different types of grains could have been important, but because of the major role they have played in shaping the solar system, gas/solid separations would seem the most likely fractionation mechanisms.

Details of the physical processes by which these separations could have occurred are complex and difficult to calculate quantitatively, but broad, qualitative outlines can be sketched. For example, if there was a stage in the gavitational collapse in which the sun was essentially totally accreted, but relatively cool, and the planetary material was widely dispersed, the Poynting-Robertson

Copyright 1991 by the American Geophysical Union.

Paper number 91GL00213

$0094-8534 / 91 / 91 \mathrm{GL}-00213 \$ 03.00$ effect could have produced a relatively large flow of solid particles to the sun. Understanding the efficiency of this is complicated by the effects of gas drag which, in turn, would depend on whether or not solid/gas fractionation had already occurred in the nebula. In the absence of nebular gas $\left(\mathrm{H}_{2}, \mathrm{He}\right)$ the grain flow could have been significant. The surface temperature of the sun is also critical. In the present-day solar system it is possible that in-falling particles are partially or totally vaporized and the products swept out either in the solar wind or by radiation pressure as a component of the so-called beta meteoroids [Grün et al, 1985]. However, even if the present sun cannot accrete mass from particles, preferential accretion of small grains could have been important in the early stages of solar evolution when surface temperatures were lower. Alternately, accretion of planetesimals in cometlike orbits could conceivably have enhanced the solid/gas ratio in the final solar composition [Joss, 1974]. The converse of this is also possible, e.g., if planetesimal formation in stable orbits was very rapid and extensive in the outer solar system, then the sun could have preferentially accreted gas rather than solids.

It is also conceivable that separation was caused by plasma effects (e.g., from intense bipolar jets) in regions where low first-ionization-potential (FIP) elements were preferentially ionized. This would impose on the whole sun the type of FIP fractionations that are now well documented between the photosphere and the solar wind [Geiss and Bochsler, 1985; Bochsler and Geiss, 1989] and solar energetic particles [Breneman and Stone, 1985]. A more speculative alternative to these scenarios would be depletion as a property of the initial interstellar cloud, reflecting long-term galactic gas/solid fractionation, e.g., due to large-scale comet formation in the galaxy [Tinsley and Cameron, 1974].

For solar system depletions the amount of dilution of late in-falling material with bulk solar matter is also an issue. If we assume that prior to $\mathrm{H}$ burning the sun was totally convecting (as opposed to the present solar state with only a surface convection zone), then the amount of excess solid material required to produce an observable solid/gas fractionation effect is much larger than for the case of preferential accretion to the sun in its present state, in which case matter would only be added to the surface convection zone. At present the mass of rockforming elements in the convection zone is about 20 Earth masses, which sets the order of magnitude of the mass requirement for preferential gain (or loss) of solids.

Comparison of the abundance of $\mathbf{K r}$ relative to nearby 
non-volatile elements in the solar wind with that estimated by interpolation from $\mathrm{CI}$ chondrite data (relative to Si) provides an observational test of preferential accretion, assuming that grain/planetesimal formation occurs under sufficiently high $\mathrm{T}$ that $\mathrm{Kr}$ was not quantitatively trapped as a solid. The $\mathrm{CI}$ interpolation is possible because of the smoothness of $\mathrm{CI}$ heavy-element abundances for odd-A nuclei [Suess, 1947; Burnett et al, 1989]. In the absence of fractionation during the formation of the sun, smoothness should permit an accurate average solar-system abundance for $\mathrm{Kr}$ to be obtained by interpolation between $\mathrm{CI}$ abundances of $\mathrm{Br}$ and $\mathrm{Rb}$. There are complications with the CI $\mathrm{Br}$ abundance [e.g., Burnett et al. 1989], but these interpolations are possible without $\mathrm{Br}$ (e.g., using Se). The probable presence of FIP depletions [e.g. Bochsler and Geiss, 1989; Steiger and Geiss, 1989] in the solar-wind $\mathrm{Kr}$ abundance is also a complication, but it does not prevent testing for preferential accretion. For example, let $\mathrm{Kr}^{*}$ be the interpolated abundance. If the solar-wind $\mathrm{K}_{\mathrm{r}} / \mathrm{Kr}^{*}$ were significantly lower than the solarwind/photospheric fractionation factors for $\mathrm{C}, \mathrm{N}$, and $\mathrm{O}$, this would be evidence for preferential accretion of solids.

Elemental abundances below Fe $(Z<26)$ are not especially smooth, so it is not possible to make the gas/solid comparison using available solar-wind $\mathrm{Ne}$ or $\mathrm{Ar}$ data with any degree of confidence. An equivalent analysis for $\mathrm{Xe}$ to that given here for $\mathrm{Kr}$ is more difficult because $\mathrm{Xe}$ corresponds to a local maximum in the solarsystem abundance curve, making interpolation difficult. In addition, it is possible that the solar-wind xenon abundance relative to the other noble gases has fluctuated significantly over the age of the solar system [Kerridge, 1980; Becker and Pepin, 1989].

\section{Solar-Wind Gases in Lunar Ilmenites}

Direct solar-wind measurements in the range of krypton are not available. The lunar regolith and gas-rich meteorites are sources of solar-wind-implanted material. However, in general solar-wind gases from these materials are fractionated relative to the solar wind itself, even in ilmenite [Eberhardt et al, 1970; 1972; Hübner et al, 1975], which appears to be the most gas-retentive phase [e.g., Frick et al, 1975]. To release potentially unfractionated solar-wind gases, it is necessary to attack the grain surfaces, where solar-wind ions are implanted, without disturbing deeper regions, where a fractionated gas reservoir is found. Frick et al [1988] suggested that this deeply-sited fractionated component derives from inward diffusion of gases previously implanted in grain surfaces, and that gases currently in the surface reservoir were implanted during the most recent exposure to the wind and thus are relatively unaltered by diffusion. They showed that lowtemperature oxidation of ilmenite from lunar soil 71501 yielded relative $\mathrm{Ne}, \mathrm{Ar}, \mathrm{Kr}$, and $\mathrm{Xe}$ abundances within $10 \%$ of "solar", as inferred by Cameron [1982], and with no evidence of systematic fractionation. An ilmenite separate from soil breccia 79035 , considered to contain solar-wind gases of greater antiquity [e.g. Kerridge, 1980], was also subjected to low temperature oxidation. Results fell within the same range with the exception of xenon, which, at -2 times higher relative to $\mathrm{Ne}, \mathrm{Kr}$, and $\mathrm{Ar}$, was suggested to indicate a secular decrease with time in the relative solar-wind xenon flux [Becker and Pepin, 1989] as suggested previously [Kerridge, 1980]. A puzzling feature of these gas releases is the nitrogen abundance, which was consistently more than an order of magnitude greater that expected for solar, and for which the authors could find no completely satisfactory explanation. One possibility [Frick et al., 1988] is that nitrogen diffuses less readily than the noble gases, so that traces of $\mathrm{N}$ from many episodes tend to accumulate in the grain surface reservoir, whereas the noble gases do not.

In spite of the possible complications pointed out in the above discussion, we will assume for the following analysis that the relative noble-gas contents from the low temperature oxidations of 71501 and 79035 ilmenites are representative of the noble-gas ratios in the solar wind.

\section{Solar-Wind ${ }^{83} \mathrm{Kr} / \mathrm{Si}$}

This is the key ratio to compare with the interpolated CI meteorite abundances, which are normalized to $\mathrm{Si}$ is necessary to calculate the solar wind ${ }^{83} \mathrm{Kr} / \mathrm{Si}$ ratio indirectly, as direct measurements are not available. Table 1 gives ${ }^{83} \mathrm{Kr} /{ }^{20} \mathrm{Ne}$ and ${ }^{83} \mathrm{Kr} /{ }^{66} \mathrm{Ar}$ ratios from the low-temperature oxidation of lunar ilmenites 71501 [Frick et $a L, 1988$ ] and 79035 [Becker and Pepin, 1989]. The ratios are multiplied by the solar-wind ${ }^{20} \mathrm{Ne} / \mathrm{Si}$ and ${ }^{36} \mathrm{Ar} / \mathrm{Si}$ ratios. To arrive at the solar-wind ${ }^{20} \mathrm{Ne} / \mathrm{Si}$ ratio, spacecraft measurements of $\mathrm{Ne} / \mathrm{O}=0.17 \pm .02$ [Bochsler and Geiss, 1989; Bochsler et al, 1986] and $\mathrm{Si} / \mathrm{O}=0.19 \pm .04$ [Bochsler, 1989] are used, along with the solar-wind neon isotopic composition of Geiss et al. [1972]. The greatest uncertainties are in the direct solar-wind measurements of $\mathrm{Ne}, \mathrm{Ar}$, and $\mathrm{Si}$. Using the higher $\mathrm{Si} / \mathrm{O}$ ratio of $0.22 \pm .07$ from Bochsler and Geiss [1989] and Bochsler [1987] would lower the estimated solar $\mathrm{Kr}$ abundance by $15 \%$. For argon the only reported solar-wind measurements are from the Apollo foil noble-gas measurements [Geiss et ah, $1972]$, from which we adopt ${ }^{36} \mathrm{Ar} / \mathrm{Ne}$. Thus, the ${ }^{36} \mathrm{Ar} / \mathrm{Si}$ ratio in the table was obtained by:

$$
\left({ }^{36} \mathrm{Ar} / \mathrm{Si}\right)_{\mathrm{Sw}}=\left({ }^{36} \mathrm{Ar} / \mathrm{Ne}\right)_{\text {foil }}(\mathrm{Ne} / \mathrm{O})_{\text {probe }} /(\mathrm{Si} / \mathrm{O})_{\text {probe }}
$$

where $\left({ }^{36} \mathrm{Ar} / \mathrm{Ne}\right)_{\text {foil }}=0.0205 \pm .0050$ [Bochsler and Geiss, 1977 and the other factors are given above. The resulting mean solar-wind ${ }^{83} \mathrm{Kr}$ abundance from Table 1 is $0.98 \pm$ $.04\left(\mathrm{Si}=10^{6}\right)$. Table 1 shows that ilmenite 79035 , which trapped more ancient solar wind, is in close agreement with the data from 71501.

The calculated solar-wind ${ }^{83} \mathrm{Kr}$ abundance of 0.98 is distinctly lower than interpolated CI abundances, e.g. 52 from Anders and Grevesse [1989]. However, the simplest interpretation of this difference is that $\mathrm{Kr}$ in the solar wind is also subject to the type of FIP or first-ionization-timo depletion already documented for $\mathrm{C}, \mathrm{N}, \mathrm{O}$, and $\mathrm{Ne}$ in the solar wind [e.g. Bochsler and Geiss, 1989] and solar-flare particles [Breneman and Stone, 1985]. These investigtions indicate that elements with FIPs greater than $\sim 11 \mathrm{eV}$ are depleted by a constant value relative to elements with lower FIPs [Steiger and Geiss, 1989]. Kr ionizes at 14.0 $\mathrm{eV}$; hence depletion of solar-wind $\mathrm{Kr}$ is expected. Applying an estimated FIP correction of $4.2 \pm 1.5$ [Steig 
TABLE 1. Data used for the calculation of the solar wind ${ }^{83} \mathrm{Kr}$ abundance. Ilmenite data from Frick et al [1988] and Becker and Pepin [1989]; solar wind ${ }^{20} \mathrm{Ne} / \mathrm{Si}$ and ${ }^{36} \mathrm{Ar} / \mathrm{Si}$ data from Geiss et aL [1972], Bochsler and Geiss [1977], Bochsler et al [1986], Bochsler [1989], and Bochsler and Geiss [1989].

\begin{tabular}{|c|c|c|c|c|c|}
\hline Sample & 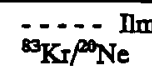 & ${ }^{80}{ }_{\mathrm{Kr}} \beta^{6 \mathrm{Ar}}$ & $\begin{array}{l}\text { Directly } \\
{ }^{20} \mathrm{Ne} / \mathrm{Si}\end{array}$ & $\begin{array}{l}\text { asured S.W. } \\
{ }^{36} \mathrm{Ar} / \mathrm{Si}\end{array}$ & $\begin{array}{l}{ }^{83} \mathrm{KI} \\
\left(\mathrm{Si}=10^{6}\right)\end{array}$ \\
\hline 71501 & $\begin{array}{l}1.137 \times 10^{6} \\
\pm .047\end{array}$ & $\begin{array}{l}5.19 \times 10^{-5} \\
\pm .34\end{array}$ & $\begin{array}{r}0.83 \\
\pm .20\end{array}$ & $\begin{array}{r}0.0183 \\
\pm .0063\end{array}$ & $\begin{array}{r}0.94 \\
\pm .23 \\
0.95 \\
\pm .33\end{array}$ \\
\hline 79035 & $\begin{array}{l}1.206 \times 10^{-6} \\
\pm .069\end{array}$ & $\begin{aligned} & 5.59 \times 10^{-5} \\
\pm & .40\end{aligned}$ & $\begin{array}{r}0.83 \\
\pm 20\end{array}$ & $\begin{array}{r}0.0183 \\
\pm .0063\end{array}$ & $\begin{array}{r}1.00 \\
\pm .25 \\
1.02 \\
\pm .36\end{array}$ \\
\hline
\end{tabular}

and Geiss, 1989; Anders and Grevesse, 1989] yields an unfractionated ${ }^{83} \mathrm{Kr}$ abundance of $4.1 \pm 1.5$, in agreement with the Cl-interpolated values in Table 2. This is shown in Figure 1 using the CI abundances of Burnett et al [1989] for which the same samples were used for all elements in this mass range.

TABLE 2. Solar wind depletion-corrected ${ }^{83} \mathrm{Kr}$ abundances from the lunar ilmenite and spacecraft data given in Table 1, along with abundance estimates from near-element $\mathrm{Cl}$ interpolations and s-process systematics.

\begin{tabular}{ll}
\hline Source & ${ }^{83} \mathrm{KJ}\left(\mathrm{Si}=10^{6}\right)$ \\
\hline Solar wind (71501 Ilmenite) & $4.0 \pm 1.4$ \\
Solar wind (79035 Ilmenite) & $4.2 \pm 1.5$ \\
Cameron [1982] & 4.7 \\
Anders and Grevesse [1989] & 5.2 \\
Burnett et al [1989]. & 4.75 \\
S-process systematics & $5.2 \pm 0.9$ \\
\hline
\end{tabular}

as quoted in Anders and Grevesse [1989]; updated from Walter et al. [1986]

For xenon, a solar abundance estimate of ${ }^{130} \mathrm{Xe}=0.24$ $\pm .09\left(\mathrm{Si}=10^{6}\right)$ is made from 71501 ilmenite data [Frick e al, 1988] using the same conversion and solar-wind fractionation factors as krypton. As mentioned earlier, interpolation of Xe from CI data is more difficult. However, calculations based on s-process systematics may be quantitatively reliable for $A \geq 100 \mathrm{AMU}$. It is interesting to note that our abundance estimate is very close to the s-process ${ }^{130} \mathrm{Xe}$ value of $0.22 \pm .09$ [Käppeler $e t$ al., 1989]. However, the xenon problem needs more study to be well understood, especially regarding its possible abundance variations with time in the solar wind.

\section{Discussion}

Within uncertainty there is no difference between our inferred solar-wind $\mathrm{Kr}$ abundance and that interpolated at mass 83 from CI meteorite data on adjacent elements. Thus, following arguments developed above, there is no evidence for gas/solid fractionation in the formation of the sun from the solar nebula. It is important to recognize the major assumption we have made in equating the relative noble-gas abundances of the solar wind with those from two lunar samples. Also, the problem with the high nitrogen to noble gas ratio in the ilmenite data must be resolved before our conclusion is unequivocal.

The propagated errors in the calculated ${ }^{83} \mathrm{Kr} / \mathrm{Si}$ are relatively large, and the lack of preferential accretion should be regarded as little better than a factor of 2 conclusion. Nevertheless, this is significant, as there are no other observational constraints on this important issue. It is important to repeat the comparison we have made with improved solar-wind data, ideally for nearby elements (Se, $\mathrm{Br}, \mathrm{Rb}, \mathrm{Sr}$ ) as well as $\mathrm{Kr}$ itself.

In hindsight it is perhaps surprising that the sun appears to have captured gas and dust from the solar nebula in approximately quantitative proportions. Conceivably, this is a crucial constraint on the early stages of solar contraction.

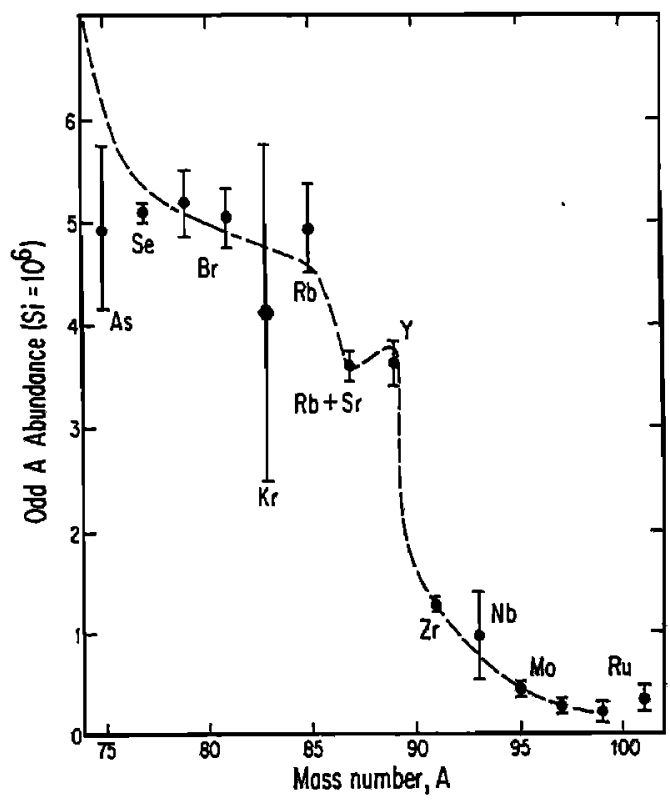

Fig. 1. Solar-system abundances of odd-mass isotopes in the range of $A=75-101$. All data except $\mathrm{Kr}$ are average CI concentrations from Burnett et al [1989], normalized to $\mathrm{Si} \equiv 10^{6}$. The $\mathrm{Kr}$ data point is averaged from the ilmenite results in Table 2, which have been corrected for photosphere/solar-wind fractionation using a value of 4.2 \pm 1.5 relative to $\mathrm{Si}$. The solar $\mathrm{Kr}$ abundance thus derived is within uncertainty of interpolation and s-process estimates, which assume no solid/gas fractionation.

Acknowledgements. This research was supported in part by NASA grants NAG 9-94 to D. Burnett and NAG 9-60 to R. O. Pepin. J. Kerridge and an anonymous reviewer are thanked for their assistance, and profitable discussions with $\mathrm{D}$. Stevenson are acknowledged. 


\section{References}

Anders, E., and N. Grevesse, Abundances of the elements: Meteoritic and solar, Geochim. Cosmochim. Acta, 53, 197-214, 1989.

Becker, R. H., and R.O. Pepin, Long-term changes in solar wind elemental and isotopic ratios: A comparison of two lunar ilmenites of different antiquities, Geochim. Cosmochim. Acta, 53, 1135-1146, 1989.

Bochsler, P., Solar wind ion composition, Physica Scripta. T18, 55-60, 1987.

Bochsler, $\mathrm{P}$., Velocity and abundance of silicon ions in the solar wind, J. Geophys. Res., 94, 2365-2373.

Bochsler, P., and J. Geiss, Elemental abundances in the solar wind, Trans. Intl. Astron. Union. XVIB. Proc. 16th General Assembly, edited by E. A. Müller and A. Jappel, pp. 120-123, D. Reidel, Dordrecht, 1977.

Bochsler, P., and J. Geiss, Composition of the solar wind, in Solar System Plasma Physics Geophysical Monograph 54, edited by J. H. Waite, Jr., J. L Burch, and R. L. Moore, pp. 133-141, 1989.

Bochsler, P., J. Geiss, and S. Kunz, Abundances of carbon, oxygen, and neon in the solar wind during the period from August 1978 to June 1982, Sol. Phys. 103. 177-201, 1986.

Breneman, H. H., and E. C. Stone, Solar coronal and photospheric abundances from solar energetic particle measurements, Astrophys. J. Lett. 294, L57-L62, 1985.

Burnett, D. S., D. S. Woolum, T. M. Benjamin, P. S. Z. Rogers, C. J. Duffy, and C. Maggiore, A test of smoothness of the elemental abundances of carbonaceous chondrites, Geochim. Cosmochim. Acta. 53, 471-481, 1989.

Cameron, A. G. W., Elemental and nuclidic abundances in the solar system, In Essays in Nuclear Astrophysics, edited by C. A. Barnes, D. D. Clayton, and D. N. Schramm, pp. 23-43, Cambridge Univ. Press, Cambridge, 1982.

Eberhardt, P, J. Geiss, H. Graf, N. Grögler, U. Krähenbühl, H. Schwaller, J. Schwarzmüller, and A. Stettler, Trapped solar wind noble gases, exposure age and $\mathrm{K} / \mathrm{Ar}$ age in Apollo 11 lunar fine material, Proc. Apollo 11 Lunar Sci. Conf. 1037-1070, 1970.

Eberhardt, P., J. Geiss, H. Graf, N. Grögler, M. D. Mendia, M. Mörgeli, H. Schwaller, A. Stettler, U. Krăhenbühl, and H. R. von Gunten, Trapped solar wind noble gases in Apollo 12 lunar fines 12001 and Apollo 11 breccia 10046, Proc. Lunar Sci. Conf. 3rd. 1821-1856, 1972.

Frick, U., H. Baur, H. Ducati, H. Funk, D. Phinney, and P. Signer, On the origin of helium, neon, and argon isotopes in sieved mineral separates from an Apollo 15 soil, Proc. Lunar Sci. Conf. 6th, 2097-2129, 1975.

Frick, U., R. H. Becker, and R. O. Pepin, Solar wind record in the lunar regolith: nitrogen and noble gases,
Proc. Lunar Planet. Sci. Conf. 18th, 87-120, Cambridge Univ. Press, Cambridge, 1988.

Geiss, J., and P. Bochsler, Ion composition in the solar wind in relation to solar abundances, In: Rapposs Isotopiques Dans le Systeme Solaire, pp. 213-240, Cepadues-Editions, Toulouse, 1985.

Geiss, J., F. Būhler, H. Cerutti, P. Eberhardt, and C Filleux, Solar wind composition experiment, Apollo 16 Preliminary Science Report, NASA SP-315, pp. 14-1 to 14-10, 1972.

Grün, E., H. A. Zook, H. Fechtig, and R. H. Giese, Collisional balance of the meteoritic complex, Icanus 62 244-272, 1985.

Hübner, W., T. Kirsten, and J. Kiko, Rare gases in Apollo 17 soils with emphasis on analysis of size and mineral fractions of soil 74241, Proc. Lunar Sci. Conf. 6th 1261-1267, 1975.

Joss, P. C., Are stellar surface heavy-element abundances systematically enhanced? Astrophys. J. 191, 771-774, 1974.

Käppeler, F., H. Beer, and K. Wisshak, S-process nucleosynthesis-nuclear physics and the classical model, Rep. Prog. Phys, 52, 945-1013, 1989.

Kerridge, J. F., Secular variations in composition of the solar wind: Evidence and causes, In Proc. Conf. Ant cient Sun, edited by R. O. Pepin, J. E. Eddy, and R. B. Merril, pp. 475-489, Pergamon, New York, 1980.

Steiger, R., and J. Geiss, Supply of fractionated gases to the corona, Astron. Astrophys. 225, 222-238, 1989.

Suess, H., Über kosmische Kernhäufigkeiten I. Mitteilung: Einige Häufigkeitsregeln und ihre Anwendung bei der Abschätzung der Häufigskeitwerte für die mittekschweren und schweren Elemente. II. Mitteilung: Einzelheiten in der Häufigskeitverteilung der mittelschweren und schweren Kerne, Z. Naturforsch. $\underline{2 a}$ 311-321, 604-608, 1947.

Tinsley, B. M., and A. G. W. Cameron, Possible influence of comets on the chemical evolution of the galaxy, Astrophys. Space Sci. 31, 31-35, 1974.

Walter, G., H. Beer, F. Käppeler, G. Reffo, and F. Fabbri, The s-process branching at ${ }^{79} \mathrm{Se}$, Astron. Astrophs. 167, 186-199, 1986.

D. S. Burnett and R. C. Wiens, Division of Geological and Planetary Sciences, California Institute of Technology, Pasadena, CA 91125.

M. Neugebauer, Mail Stop 169-506, Jet Propulsion Laboratory, Pasadena, CA 91109.

R. O. Pepin, School of Physics and Astronomy, University of Minnesota, Minneapolis, MN 55455.

(Received August 17, 1990; revised November 19, 1990; accepted November 21,1990 ) 